\title{
Metodologias sensoriais descritivas mais rápidas e versáteis - uma atualidade na ciência sensorial
}

\author{
Rapid and versatile sensory descriptive methods - an updating of sensory science
}

\author{
Marcela de Alcantara ${ }^{1 *}$, Daniela De Grandi Castro Freitas-Sá \\ 1 Universidade Federal Rural do Rio de Janeiro (UFRRJ), Departamento de Tecnologia de Alimentos, Seropédica/RJ - Brasil \\ ${ }^{2}$ Empresa Brasileira de Pesquisa Agropecuária (EMBRAPA), Agroindústria de Alimentos, Laboratório de Análise Sensorial e Instrumental, Rio de \\ Janeiro/RJ - Brasil
}

\section{*Corresponding Author}

Marcela de Alcantara, Universidade Federal Rural do Rio de Janeiro (UFRRJ), Departamento de Tecnologia de Alimentos, Rodovia BR 465, km 07, s/n, Zona Rural, CEP: 23890-000, Seropédica/RJ - Brasil, e-mail: marceladealcantara@gmail.com

Cite as: Rapid and versatile sensory descriptive methods - an updating of sensory science. Braz. J. Food Technol., v. 21, e2016179, 2018.

Received: Dec. 05, 2016; Accepted: Sept. 27, 2017

\section{Resumo}

Os métodos sensoriais descritivos permitem a detecção, a descrição e a quantificação dos atributos sensoriais presentes em um alimento. Estes métodos são utilizados pela indústria de alimentos no desenvolvimento de novos produtos, no controle de qualidade, nas alterações de ingredientes e/ou formulações e na avaliação de produtos durante a estocagem. Porém, a maioria das técnicas descritivas existentes necessita da utilização de avaliadores treinados e emprega uma escala não estruturada para avaliar os produtos. Isto torna as análises demoradas e com custo elevado, devido às exaustivas sessões de treinamento para que os problemas com o uso das escalas sejam minimizados. Com o intuito de reduzir o tempo de análise e os custos inerentes aos testes descritivos, pesquisas recentes têm buscado desenvolver metodologias que permitam a descrição rápida dos alimentos e que possam também ser aplicadas com consumidores. Este trabalho teve como objetivo abordar os progressos da ciência sensorial quanto ao desenvolvimento de novas metodologias descritivas mais rápidas e versáteis.

Palavras-chave: Perfil livre; Perfil flash; Sorting; Mapeamento projetivo; Check-all-that-apply; Posicionamento sensorial polarizado.

\section{Abstract}

Sensory descriptive methods allow for the detection, description and quantification of the sensory attributes present in a food. These methods are used by the food industry to develop new products, for quality control, to change ingredients and/or formulations, and in food storage studies. However, most existing descriptive techniques require the use of trained assessors and rate using unstructured scales to evaluate the foods. This makes the analysis time longer and the analysis cost higher due to the extensive training sessions in order to minimize the problems caused by the use of the scales. In order to reduce the analysis time and costs inherent in the descriptive tests, recent research has aimed to develop methodologies that allow for the rapid description of foods and that can also be applied with consumers. The aim of this study was to consider the progress made in sensory science concerning the development of new faster and more versatile descriptive methodologies.

Keywords: Free choice profile; Profile flash; Free sorting task; Projective mapping; Check-all-that-apply; Polarized sensory positioning.

\section{Introdução}

As técnicas descritivas são métodos abrangentes e flexíveis, capazes de fornecer informações detalhadas sobre as propriedades sensoriais de um alimento, constituindo-se em uma das mais importantes ferramentas da análise sensorial. Quando utilizadas em conjunto com testes de consumidor, fornecem importantes informações para introdução e 
posicionamento estratégico de produtos no mercado (LAWLESS; HEYMANN, 1999), pois permitem identificar os atributos sensoriais que dirigem a preferência do consumidor.

As limitações que merecem atenção são o tempo necessário para treinamento dos avaliadores, a definição de materiais de referência que possam traduzir as percepções ou sensações, e o limitado escopo do vocabulário, que pode não ser suficiente para definir a qualidade sensorial do alimento. Considerando-se o aspecto econômico e o tempo consumido para treinamento da equipe de avaliadores, novos estudos vêm apresentando métodos mais versáteis para atender às necessidades da indústria de alimentos. Nos últimos 20 anos, houve um importante progresso na ciência sensorial concernente ao desenvolvimento de novas metodologias, para uma rápida descrição dos produtos, e avanços no entendimento sobre as respostas de diferentes segmentos de consumidores.

Muitos dos estudos mais recentes (ANTÚNEZ et al., 2017; LAZO et al., 2016; VIDAL et al., 2016; FLEMING et al., 2015; CADENA et al., 2014; BRUZZONE et al., 2012; DEHLHOLM et al., 2012) comparam as técnicas de perfil sensorial aplicadas nos consumidores com a análise descritiva clássica. Diversos tipos de produtos já foram utilizados, sendo que as mesmas amostras são analisadas pela equipe treinada e por grupos de consumidores. A comparação se dá por índices estatísticos específicos e comparação do mapa sensorial gerado.

Esta revisão tem como objetivo abordar a teoria, as vantagens e desvantagens, e discutir recomendações para a aplicação das novas técnicas de perfis sensoriais desenvolvidas nos últimos anos.

\section{Análise descritiva quantitativa}

A Análise Descritiva Quantitativa $\left(A D Q{ }^{\circledR}\right)$ é a técnica de descrição sensorial mais utilizada na área de alimentos, pois permite o levantamento, a descrição e a quantificação dos atributos sensoriais detectáveis no produto, utilizando avaliadores com alto grau de treinamento e uma análise estatística robusta dos dados (STONE; SIDEL, 2004).

Esta metodologia envolve três etapas fundamentais: i) o levantamento de atributos e a familiarização dos avaliadores com os produtos; ii) a definição, em consenso com a equipe de avaliadores, dos termos descritores e a fixação das referências que servirão como padrões de intensidade (mínima e máxima) para cada atributo, e iii) a avaliação das amostras utilizando, normalmente, uma escala não estruturada de $9 \mathrm{~cm}$ para quantificação da intensidade dos atributos sensoriais. Os dados gerados são transformados em médias de intensidade, por atributo, e avaliados estatisticamente por Análise de Variância (ANOVA). Nos casos em que diferenças entre as amostras são encontradas, testes de comparação de médias, usualmente o Teste de Tukey, são realizados.
A equipe é frequentemente monitorada com relação ao seu desempenho (concordância, capacidade discriminativa, repetibilidade) e à reprodutibilidade, individual ou do grupo como um todo, durante os treinamentos, com o objetivo de alcançar resultados mais precisos e, portanto, mais confiáveis e consistentes (NBR ISO 11132 ABNT, 2016; MOUSSAOUI; VARELA, 2010).

No entanto, criar e manter uma equipe sensorial bem treinada pode ser muito caro. A etapa de seleção dos avaliadores pode ser relativamente longa, variando entre 10 e 120 horas, e o número de sessões ou o tempo de treinamento está relacionado à complexidade do produto, o que pode ser restritivo, particularmente quando se exigem respostas rápidas ao mercado (LAWLESS; HEYMANN, 2010). Diante disso, surgiu o método Perfil Livre (WILLIAMS; LANGRON, 1984), pelo qual cada avaliador desenvolve uma lista própria de atributos e definições para avaliação das amostras, praticamente eliminando a etapa de treinamento da equipe. Essa técnica foi descrita pela primeira vez para a avaliação de vinhos e baseia-se no princípio de que as pessoas percebem as mesmas características no produto, mesmo que se expressem de forma diferenciada (OLIVEIRA; BENASSI, 2003).

A técnica estatística que é aplicada aos dados obtidos com o Perfil Livre é a Análise de Procrustes Generalizada (GPA). O princípio da análise é aproximar as configurações de cada avaliador a uma configuração consenso, maximizando as similaridades geométricas (GOWER, 1975). Os resultados de cada avaliador são considerados como coordenadas num espaço multidimensional, que são transformadas de maneira a evitar variação no uso da escala, diferentes intervalos de valores ou interpretações diferenciadas dos atributos (DIJKSTERHUIS; GOWER, 1991). Como desvantagem, o total da variância explicada por uma solução bidimensional é menor do que a observada na análise descritiva quantitativa, o que pode ser atribuído aos avaliadores diferirem mais, por não terem sido treinados. Por isso, deve-se estudar a possibilidade de utilizar um número maior de dimensões para explicação das variáveis, conforme as soluções tridimensionais apresentadas em alguns resultados (KOBAYASHI; BENASSI, 2012; TERHAAG; BENASSI, 2010).

Em resposta às limitações da análise descritiva clássica, várias metodologias vêm sendo estudadas como alternativa à análise descritiva convencional, dentre as quais são abordadas no presente trabalho, as técnicas Perfil Flash, sorting, mapeamento projetivo, Check-All-That-Apply e posicionamento sensorial polarizado. Estas metodologias não necessitam de treinamento e podem ser realizadas por avaliadores treinados ou não treinados. São baseadas: i) em descrições verbais de produtos, permitindo que os avaliadores usem linguagem própria na descrição dos atributos; ii) na semelhança ou diferença entre os produtos (a verbalização destas 
diferenças ocorre apenas em uma segunda etapa ou tais diferenças podem mesmo ser omitidas), e iii) na comparação dos produtos individualmente ou com um conjunto de referências (VALENTIN et al., 2012).

\section{Novas metodologias descritivas}

\subsection{Perfil Flash}

A metodologia Perfil Flash surgiu de uma modificação proposta por Dairou e Siefferman (2002) e está baseada numa combinação do método de Perfil Livre com a técnica de ordenação. Assim como no Perfil Livre, o avaliador desenvolve seu próprio vocabulário para descrever os atributos sensoriais pertinentes ao produto. Porém, em vez de determinar a sua intensidade através do uso de escalas não estruturadas, o avaliador ordena os produtos comparados, reduzindo assim o número de sessões (TERHAAG; BENASSI, 2010). A apresentação do conjunto de amostras para os avaliadores é simultânea, o que permite, então, avaliar as amostras comparativamente. Isto traz consequências práticas importantes, principalmente em termos de preparo e apresentação das amostras.

Os avaliadores são instruídos a provar as amostras, a fim de descrevê-las, usando quaisquer atributos não hedônicos que considerem apropriados, desde que suficientemente discriminativos para permitir uma classificação das amostras ou mesmo uma classificação parcial. Eles são convidados a descrever todas as diferenças percebidas e não há orientação quanto ao número de atributos a serem utilizados. Em alguns casos, o profissional sensorial pode decidir restringir o estudo a apenas alguns aspectos - por exemplo, textura ou sabor - e as instruções são, então, adaptadas em conformidade. Os avaliadores devem então classificar imediatamente todas as amostras para cada atributo concebido, na ordem da sensação mais fraca até a mais forte. Geralmente, recomenda-se que as duas etapas - atribuição dos descritores e classificação - sejam realizadas conjuntamente (DELARUE, 2014). No entanto, alguns avaliadores acham mais conveniente listar todos os atributos que desejam avaliar e depois classificar as amostras.

Como não há treinamento ou discussão com a equipe, o procedimento pode ser individualizado e sintetizado em apenas uma sessão, de maneira a permitir equipes maiores, com uma abordagem mais próxima de estudos de consumidor. Estudos que utilizaram esta técnica, em geral, trabalharam com equipes experientes e constatam maior rapidez e facilidade de execução das análises, com redução de custos pertinentes à quantidade de amostra utilizada (DELARUE; SIEFFERMANN, 2004; TAREA et al., 2007), já que as fases de familiarização, geração e avaliação são integradas em uma única etapa. Como desvantagem, observa-se que a interpretação dos termos sensoriais nem sempre é fácil, devido ao grande número de termos e à falta de definições e procedimentos de avaliação (ALBERT et al., 2011). Além disso, não é possível quantificar, mas sim apenas identificar as diferenças entre as amostras analisadas.

A tarefa de avaliação simultânea limita o número de amostras que podem ser avaliadas em uma única sessão, mas esta decisão depende mais da natureza dos produtos a serem avaliados. Segundo Delarue (2014), o analista deve considerar as restrições de obtenção e disponibilidade do produto, além da viabilidade de se apresentarem todas as amostras nas mesmas condições (quantidade, temperatura, etc.). No entanto, deve-se ponderar que a forma como uma sessão é conduzida no Perfil Flash é flexível e pode ser projetada para tornar a tarefa de avaliação viável, planejando intervalos entre atributos ou sessões separadas de dois ou três atributos, somente.

A técnica pode ser aplicada também se utilizando consumidores; no entanto, orientações sobre o procedimento devem ser fornecidas com exemplos muito didáticos. O tamanho da equipe frequentemente utilizada é de cerca de 40 a 50 consumidores, embora, dependendo do objetivo do estudo, trabalhos apresentem a técnica aplicada com consumidores variando de 24 (MOUSSAOUI; VARELA, 2010) a 200 participantes (BALLAY et al., 2006). Montanuci et al. (2015) avaliaram sensorialmente amostras de sucos de maracujá aplicando o Perfil Flash a 89 consumidores. As amostras possuíam diferentes quantidades de açúcares, espessantes e corantes. Segundo os autores, os consumidores demonstraram boa capacidade em discriminar e descrever os produtos analisados.

Como os atributos avaliados pelos avaliadores não são supostamente os mesmos, não há maneira de visualizar dados por meio da pontuação média ou equivalente. Em vez disso, as técnicas de análise de dados multivariadas são utilizadas. Assim como no Perfil Livre, a técnica estatística aplicada aos dados obtidos é a Análise de Procrustes Generalizada (GPA) (DEHLHOLM et al., 2012). A técnica de análise fatorial múltipla (AFM) também pode ser aplicada (ESCOFIER; PAGÈS, 2008). Essas duas técnicas produzem mapas fatoriais que podem ser usados para avaliar o posicionamento sensorial dos produtos.

\subsection{Sorting}

Este método consiste na classificação de produtos em grupos, de acordo com suas semelhanças ou diferenças, utilizando-se critérios individuais do avaliador. Todas as amostras são apresentadas simultaneamente, dispostas em uma mesa em ordem randomizada, para cada avaliador. Os avaliadores são solicitados a olhar, cheirar e/ou provar - dependendo dos objetivos do estudo - todos os produtos e depois classificá-los em grupos contraditórios, com base nas semelhanças percebidas entre eles. Os avaliadores são livres para criar quantos grupos acharem necessários, 
classificando quantas amostras quiserem, em cada grupo. Após a separação dos grupos, os avaliadores podem fornecer alguns termos para caracterizar cada grupo formado. Na área alimentícia, o sorting já foi aplicado em diversos produtos, como queijos, água potável, geleias de frutas, cervejas, vinhos, iogurtes, condimentos, pepinos e tomates, azeite de oliva e carne (CHOLLET et al., 2014).

Para analisar os dados da classificação, os resultados de cada avaliador são codificados numa matriz de co-ocorrência individual, na qual os produtos avaliados compõem as linhas e as colunas. Um valor de '1' na interseção de uma linha e uma coluna indica que o avaliador classificou esses dois produtos juntos, enquanto que um valor de ' 0 ' indica que os produtos não foram reunidos. Todas as matrizes individuais são então resumidas para obter uma matriz de similaridade global. A matriz de similaridade é geralmente analisada por escalonamento multidimensional (MDS), uma técnica usada para visualizar a proximidade ou a distância entre objetos, em um espaço de baixa dimensão. No MDS, cada objeto é representado por um ponto em um mapa, posicionando assim os produtos em uma representação visual através de algoritmos, que podem ser classificados como métrico ou não métrico (ABDI, 2007a,b; ABDI; WILLIAMS, 2010).

A princípio, o sorting não apresenta restrição quanto ao tipo de avaliadores. Alguns estudos mostram que avaliadores treinados e não treinados apresentam mapas sensoriais perceptivos semelhantes, como nas avaliações de cereais matinais (CARTIER et al., 2006) e cervejas (CHOLLET et al., 2011). Outros relatam haver diferenças entre os mapas derivados da classificação por sorting e pela análise descritiva clássica, sendo que, normalmente, o primeiro apresenta uma discriminação ligeiramente menor entre as amostras (LELIĖVRE et al., 2008). Para incentivar uma discriminação mais refinada, Santosa et al. (2010) propuseram uma modificação no sorting, ao avaliarem amostras de azeite de oliva extra virgem, promovendo a tarefa de classificação em dois estágios e, assim, a criação de subgrupos dentro dos grupos previamente criados, o que permitiu que os avaliadores utilizassem mais critérios ou visões multidimensionais de sua percepção dos produtos.

Embora a metodologia seja considerada de fácil entendimento e agradável aos avaliadores - treinados ou não - por utilizar-se de uma atividade mental natural (COXON, 1999), quando aplicada com consumidores, pode apresentar alguma dificuldade na execução dos testes, conforme relatado por Chollet et al. (2011), que avaliaram a eficiência da técnica do sorting utilizando seis conjuntos distintos de cervejas. Foi realizada uma série de estudos destacando as vantagens e delineando os limites da técnica: número de amostras, número de avaliadores, influência do treinamento dos avaliadores e do direcionamento do teste, como o fornecimento de uma lista de atributos ou definindo uma quantidade de grupos a ser formada. Os autores concluíram que a ferramenta necessita de um número maior de avaliadores do que a metodologia convencional, limitada a um número mínimo de 20 avaliadores não treinados, no caso de cervejas. Como as amostras são apresentadas simultaneamente em sessão única, quando se trata de produtos complexos e fatigantes, a quantidade de amostras é um fator limitante. Outro fator limitante a ser avaliado pelo profissional sensorial é a necessidade de manutenção da temperatura que alguns produtos requerem ao serem apresentados, visto que o tempo utilizado para realizar a tarefa de classificação no sorting é muito variável entre os avaliadores.

\subsection{Mapeamento projetivo}

Vários autores compararam recentemente o mapeamento projetivo, bem como outros métodos descritivos rápidos, com a técnica de perfil convencional, destacando que a técnica foi aplicada com êxito para vários fins (DEHLHOLM et al., 2012; VALENTIN et al., 2012, VARELA; ARES, 2012). O princípio do mapeamento projetivo é semelhante ao sorting, diferindo na tarefa de classificação, na qual os avaliadores são solicitados a posicionar as amostras em uma superfície plana, como, por exemplo, uma folha de papel. Os avaliadores são instruídos a posicionar amostras percebidas como semelhantes mais próximas umas das outras, enquanto que as amostras menos similares são colocadas mais afastadas. Desta forma, todas as amostras são posicionadas com relação umas às outras. Normalmente, os avaliadores decidem sobre os próprios critérios de discriminação e é comum obter informações descritivas dos produtos, adicionando algumas palavras que descrevem cada amostra.

A superfície utilizada para posicionar as amostras pode apresentar distintas formas: retângulo, quadrado, círculo e elipse (DEHLHOLM, 2014). Hopfer e Heymann (2013), ao avaliarem a influência do formato da superfície, perceberam que o espaço fornecido influencia na representação dos produtos e que os avaliadores tendem a usar mais o eixo horizontal do que o vertical. Isto indica que uma configuração quadrangular pode levar a uma menor discriminação das amostras. Em relação ao tamanho da superfície, nenhum estudo verificou se existe uma relação entre diferentes tamanhos de uma mesma geometria. No entanto, diferentes formatos A4, A3 (RISVIK et al., 1994, 1997), 60 cm × 40 cm (PAGÈS, 2005), $60 \mathrm{~cm} \times 60 \mathrm{~cm}($ KENNEDY; HEYMANN, 2009; NESTRUD; LAWLESS, 2010), e 40 cm de diâmetro (DEHLHOLM, 2012) já foram utilizados por diferentes autores.

As distâncias entre amostras no mapeamento projetivo são geralmente tratadas por modelos algoritmos, como a distância euclidiana, e significam as diferenças entre as amostras posicionadas de acordo com as instruções de avaliação, ou seja, produtos mais próximos, mais similares, 
e produtos mais distantes, mais diferentes. Mesmo quando as instruções inicialmente podem parecer claras, há variação na maneira pela qual os avaliadores as interpretam. Vários autores sugeriram que as estratégias de projeção podem variar de avaliador para avaliador (DEHLHOLM, 2012; HOPFER; HEYMANN, 2013), dependendo do tipo e do nível de treinamento. Portanto, é recomendado que o profissional sensorial dedique mais tempo para introduzir o método para avaliadores iniciantes, de modo que toda a área da projeção seja utilizada. As estratégias de projeção observadas em avaliadores iniciantes foram: linear, projeção em L, projeção em T, dispersa (a qual ocupa uma grande área da geometria delimitada), irregular (quando todos os produtos são colocados juntos em uma pequena área da geometria delimitada), categórica (mostrando claramente agrupamentos de produtos distantes uns dos outros, aleatoriamente) e categórica bilinear (observada como uma combinação de dois padrões lineares claramente separados).

As coordenadas cartesianas $(x, y)$ de cada posicionamento das amostras e as frequências dos descritores de amostras semelhantes constituem as duas matrizes estruturais de dados que são analisadas. Uma vez que os avaliadores podem ter utilizado critérios diferentes para diferenciar as amostras, uma análise que leva em conta conjunto de dados variados, como a análise fatorial múltipla (AFM), é desejável, incluindo a matriz de descritores como um grupo de variável suplementar na análise. Nos dados de posicionamento, ou seja, duas variáveis por avaliador, sendo uma a coordenada $x$ e outra a coordenada $y$, a análise AFM é dada em duas etapas, sendo a primeira uma análise de componentes principais de cada conjunto de variáveis, a menos que os dados sejam categóricos, o que seria uma análise de correspondência múltipla. O segundo passo é uma nova análise de componentes principais sobre os resultados concatenados, que termina com a construção de uma configuração global (ABDI et al., 2013).

Como as amostras são projetadas em um espaço 2D, o número mínimo de amostras a ser considerado deve ser quatro, número necessário para cobrir as dimensões (DEHLHOLM, 2014). No entanto, estudos abordam uma quantidade maior de amostras, variando de cinco (PAGÈS, 2005) a 18 amostras (KING et al., 1998). Quanto ao número de avaliadores, não há estudos que tenham concluído acerca de um número mínimo necessário. Estudos mostram uma variação de oito (PAGÈS, 2005; KENNEDY; HEYMANN, 2009) a 83 indivíduos (DEHLHOLM, 2012), sendo que esta definição está mais relacionada com a natureza do conjunto de amostras e com o tipo de avaliador. Em se tratando de avaliadores não treinados, Vidal et al. (2014) forneceram uma visão sobre o número de consumidores necessário para alcançar configurações sensoriais estáveis. Nos 21 conjuntos de dados analisados, os resultados sugerem que, quando se trabalha com amostras muito diferentes, 50 consumidores parece ser uma recomendação segura do número mínimo para obter resultados confiáveis, com mapeamento projetivo. No entanto, após a conclusão de qualquer caracterização por mapeamento projetivo, é altamente recomendável verificar a confiabilidade da configuração do espaço de amostra, usando procedimentos estatísticos apropriados. Os resultados mostraram que a estabilidade da configuração depende claramente do grau e do tipo de diferença entre as amostras, e do número de amostras no conjunto de dados.

E, ao comparar a performance de avaliadores treinados e consumidores na utilização desta técnica, alguns autores sugerem que ambos concordam na maioria dos aspectos sensoriais das amostras analisadas; no entanto, a equipe treinada é capaz de identificar e descrever pequenas diferenças sensoriais que podem não ser percebidas por consumidores (BARCENAS et al., 2004; KENNEDY; HEYMANN, 2009).

Para obter mais informações dos avaliadores, Dehlholm (2014) propôs uma modificação denominada mapeamento projetivo dirigido, no qual o posicionamento dos produtos é feito de acordo com uma categoria de atributos direcionada (aparência, textura), diferente da avaliação global. Esta técnica é referida como perfil ultra flash por Perrin et al. (2008).

\subsection{Check-all-that-apply}

A metodologia Check-All-That-Apply (CATA) é a técnica que mais vem sendo utilizada para coletar informações sobre a percepção dos consumidores sobre as características sensoriais dos produtos. O formato da questão CATA permite aos consumidores escolher todos os atributos possíveis para descrever o produto, a partir de uma lista apresentada. De acordo com Dooley et al. (2010), os termos desta lista podem ser gerados por um painel de avaliadores treinados ou por um grupo de consumidores, ao testar o produto (por exemplo, em um grupo focal). Além disso, os descritores não são limitados aos atributos sensoriais do produto, mas também podem estar relacionados ao uso do produto ou ao conceito em que se encaixam. Como as respostas CATA estão diretamente ligadas à percepção dos consumidores das características do produto, essas respostas podem ser utilizadas como dados suplementares, para maximizar a aceitação dos produtos.

Ares et al. (2014a) investigaram a reprodutibilidade do questionário CATA na caracterização sensorial de diferentes produtos, através da introdução de uma segunda sessão de avaliação. Em todos os estudos, os mesmos consumidores avaliaram o mesmo conjunto de amostras sob condições idênticas, nas duas sessões. O estudo revelou que a técnica mostrou-se, de uma forma altamente reprodutível, bastante capaz de detectar diferenças e 
caracterizar os diferentes produtos, confirmando pesquisas anteriores (JAEGER et al., 2013; BRUZZONE et al., 2012; LADO et al., 2010).

A metodologia CATA é descrita como eficiente para descrever e discriminar os produtos, sendo suas principais vantagens a simplicidade, e a rapidez com que as análises são efetuadas. Estudos que comparam sua eficiência em relação ao uso de avaliadores treinados relatam altas correlações detectadas entre as avaliações, evidenciando que os consumidores são capazes de avaliar os atributos sensoriais de uma forma semelhante (BRUZZONE et al., 2012; ARES et al., 2010). Sua principal limitação, segundo Dolley et al. (2010), reside no fato de não serem medidas as intensidades dos atributos apresentados na lista. Por causa disso, em comparação com outras técnicas, pode apresentar menor poder de discriminação (ANTÚNEZ et al., 2017), principalmente quando as amostras possuem diferenças sutis nas intensidades dos atributos. A discriminação entre as amostras é verificada através da aplicação do teste estatístico Q de Cochran, amplamente utilizado nos dados de frequência dos atributos da lista CATA, para inferência das diferenças dos produtos por atributo (CASTURA; MEYNERS, 2014). Além disso, a técnica requer um grande número de consumidores. Embora não haja investigação suficiente que indique um número adequado de participantes, um estudo com relevância estatística deve provavelmente considerar o uso de 100 consumidores ou mais.

A problemática da medição da intensidade gerou modificações na forma de aplicação e condução do CATA, como proposto pela metodologia Rate-all-that-apply (RATA), na qual os consumidores são solicitados a indicar, além dos termos apropriados para descrever o produto, a intensidade dos termos selecionados (REINBACH et al., 2014); e pela Temporal Check-All-That-Apply (TCATA), que mede a percepção sensorial ao longo de um tempo pré-determinado, além do TCATA fading, em que os termos são automaticamente desmarcados após um período de tempo pré-determinado, e os avaliadores devem re-selecioná-los se eles ainda os consideram apropriados para descrever a amostra analisada. (ARES et al., 2014b, 2015c; CASTURA et al., 2016; VIDAL et al., 2016).

Outro fato que deve ser observado é a escolha e o número dos termos que irão fazer parte do questionário CATA. Hughson e Boakes (2002) mostraram que o fornecimento de uma lista curta, em vez de uma lista maior, para descrever um conjunto de vinhos, levou a descrições mais eficientes. Em estudo mais abrangente, Jaeger et al. (2015) analisaram sete estudos de consumidor envolvendo um total de 735 indivíduos e cinco categorias diferentes de produtos (biscoito, queijo, bebidas de frutas, chocolate e sobremesa láctea). O uso de listas "curtas" ou "longas" (10 a 17 termos versus 20 a 28 termos) teve pouco impacto na caracterização sensorial dos produtos.
No entanto, os resultados apontaram para um efeito de "diluição" na frequência de citação quando "listas longas" criadas usando palavras sinônimas/antônimas foram utilizadas, confirmando as expectativas de idiossincrasia na percepção do consumidor ou a redução da capacidade discriminativa da lista de termos. Os autores concluíram que, ao conceber os termos da lista, os profissionais devem, em vez de utilizar um número excessivo de termos, incluir termos diferentes que façam referência às características sensoriais relevantes, a fim de reconhecer a heterogeneidade do consumidor. Ares e Jaeger (2013) demonstraram que a ordem em que os termos são incluídos em um questionário CATA influencia a resposta do consumidor. Segundo os autores, os atributos localizados mais próximos ao topo da lista tendem a ser mais utilizados. Em um estudo subsequente, Ares et al. (2015d) recomendam que a ordem de apresentação dos termos no questionário seja balanceada inter e intra participantes, minimizando a influência de vieses sobre as respostas dos consumidores e mantendo sua atenção ao longo da tarefa. Porém, Meyners e Castura (2016) sugerem que os benefícios do balanceamento entre os avaliadores superam os benefícios do balanceamento intra-avaliadores. Neste sentido, recomendam a atribuição de ordens de atributo aos avaliadores em vez de atribuir às amostras, tanto no questionário CATA quanto na técnica de domínio temporal de sensações (TDS) e TCATA. Estudos recentes exploram abordagens diferentes no uso da metodologia CATA, em busca da inovação no desenvolvimento de produtos. Ares et al. (2017) utilizaram o questionário CATA para identificar como os produtos diferem do produto ideal esperado pelos consumidores, incluindo termos no questionário CATA com conotações de intensidade hedônica (por exemplo, não suficientemente doce, doce demais) e aplicando o questionário CATA para caraterização dos produtos experimentados e dos produtos ideais (ou idealizados). Jaeger et al. (2017) investigaram se contextos evocados influenciam na discriminação hedônica e na caracterização sensorial de produtos, usando o questionário CATA. Vidal et al. (2016) apresentaram uma comparação exaustiva entre as metodologias CATA e RATA, com relação ao uso dos termos, à discriminação e às configurações sensoriais das amostras. Meyners (2016) propôs uma modificação na análise de dados da técnica de domínio temporal de sensações (TDS), agregando o formato de questões CATA em intervalos de tempo específicos para realçar as relações entre atributos e respostas hedônicas, de modo a considerar a dimensão temporal das respostas. Ruark et al. (2016) investigaram a percepção de consumidores mais velhos e o desempenho da variante Yes/No forced-choice Check-All-That-Apply with ideal (CATA-I), em comparação ao Método do Perfil Ideal em testes conduzidos nos lares, com base na facilidade de uso, no tempo necessário para completar a tarefa e na capacidade de discriminação dos 
produtos. Antúnez et al. (2016) apresentaram considerações adicionais sobre esta metodologia, explorando as diferenças individuais na atenção visual dada à lista CATA, por meio de um equipamento remoto para gravar os movimentos oculares dos participantes.

\subsection{Posicionamento sensorial polarizado}

O Posicionamento Sensorial Polarizado (PSP) é baseado na comparação das amostras com um conjunto de referências fixas, denominadas pólos (TEILLET, 2014). O princípio desta técnica é basicamente avaliar a similaridade global (ou dissimilaridade) entre amostras e cada um dos pólos, estrategicamente escolhidos dentre as categorias do produto. Para seleção dos pólos, devem-se levar em consideração as principais características sensoriais responsáveis pelas semelhanças e diferenças entre as amostras. Diferentes abordagens podem ser usadas para coletar informações sobre as semelhanças (ou dissimilaridades) entre amostras e pólos. A primeira coleta refere-se diretamente às diferenças com relação aos pólos, pedindo aos avaliadores que pontuem a dissimilaridade global em uma escala contínua, variando de "exatamente igual" a "totalmente diferente" (TEILLET et al., 2010). A segunda coleta propõe deduzir semelhanças e dissimilaridades dos pólos, pedindo que os avaliadores indiquem a qual dos pólos cada amostra é mais semelhante. A utilização de amostras como referência permite obter dados em diferentes sessões, o que se torna a principal vantagem da metodologia, quando se tem por objetivo a caracterização sensorial de um conjunto muito grande de amostras ou quando há amostras com características complexas para serem avaliadas (ARES; VARELA, 2014).

Inicialmente desenvolvida para a caracterização sensorial de águas minerais (TEILLET et al., 2010), a metodologia já foi utilizada em alguns produtos, como queijo e almôndegas (VARELA et al., 2014), iogurte (CADENA et al., 2014), bases para pele e suco em pó sabor laranja (DE SALDAMANDO et al., 2013), bebidas lácteas sabor chocolate, sobremesa láctea sabor baunilha e suco em pó sabor laranja (ARES et al., 2015b).

A seleção dos pólos é provavelmente o passo mais importante para a aplicação desta metodologia. Neste sentido, as questões essenciais que surgem são definir a quantidade de pólos e determinar quais produtos a serem utilizados como tal. Segundo Ares et al. (2015a), o aumento do número de pólos torna a tarefa mais difícil e tediosa, aumentando a fadiga sensorial. O número habitual de pólos, utilizado em estudos com o PSP, é três (ANTÚNEZ et al., 2015; ARES et al., 2015b; CADENA et al., 2014; DE SALDAMANDO et al., 2013; TEILLET, 2014). Este número tem sido recomendado assumindo-se que a maioria das informações sobre as características sensoriais das amostras seria representada num espaço bidimensional e considerando-se que três pólos seriam necessários para estabilizar esse espaço.

De Saldamando et al. (2013) utilizaram o PSP para estudar a influência de conjuntos de três pólos com características distintas sobre a caracterização de produtos. Os resultados forneceram evidências preliminares sobre a estabilidade dos espaços sensoriais obtidos com um conjunto diferente de pólos (coeficientes RV superiores a $0,908 ; p<0,05)$. No entanto, foram identificadas diferenças nas conclusões sobre as semelhanças e diferenças entre as amostras, dependendo do conjunto de pólos que foi utilizado, o que sugere a necessidade de selecionar cuidadosamente pólos estáveis.

Ares et al. (2015b) investigaram a influência do número de pólos utilizados e das características desses pólos na descrição das amostras. Ao final das avaliações, os consumidores indicaram como eles avaliaram o grau de diferença entre as amostras e os pólos e enumeraram as características sensoriais dos pólos que tomaram em consideração para a avaliação. Concluiu-se que os consumidores tendem a avaliar o grau de diferença entre as amostras e cada um dos pólos avaliando a intensidade de uma ou duas características importantes, e que é possível obter um espaço sensorial multidimensional com a utilização de apenas dois pólos, especialmente quando os pólos representam claramente as características sensoriais responsáveis pelas principais diferenças entre as amostras.

\section{Comparação entre os métodos descritivos}

A similaridade entre dois conjuntos de variáveis que foram medidos, a partir de um mesmo conjunto de amostras, pode ser verificada através da análise visual dos mapas sensoriais gerados, da análise fatorial múltipla (AFM) ou através do coeficiente RV (ALBERT et al., 2011; ANTÚNEZ et al., 2017; ARES et al., 2011; CADENA et al., 2014; FLEMING et al., 2015; LAZO; CLARET; GUERRERO, 2016). Em estudos recentes, o coeficiente $R V$ vem sendo o mais utilizado para medir esta similaridade. É obtido através de comandos em softwares estatísticos e, conceitualmente, é um coeficiente de correlação multivariada. Esse coeficiente toma valores entre ' 0 ' e ' 1 ', em que ' 1 ' representa a similaridade perfeita. Alguns comandos estatísticos também fornecem o teste de significância do coeficiente RV (DEHLHOLM, 2014), o que indica a validade de suas conclusões. Porém, muitas vezes, os valores de significância do coeficiente RV não são apresentados.

Albert et al. (2011) realizaram o perfil sensorial de amostras de nuggets de peixe, utilizando a metodologia convencional (ADQ), o Perfil Flash (PF) e o mapeamento projetivo (MP). A ADQ foi realizada com 10 avaliadores treinados; o Perfil Flash com 10 avaliadores com experiência em análises descritivas em outros produtos e o mapeamento projetivo com 20 consumidores. As três metodologias 
apresentaram resultados similares, mostrando que o Perfil Flash (ADQ vs. PF, RV = 0,856; p-valor não apresentado) e o mapeamento projetivo (ADQ versus $M P, R V=0,816$; p-valor não apresentado) podem ser utilizados como uma alternativa rápida para descrição de alimentos que necessitam ser consumidos em altas temperaturas, em substituição a $A D Q$, que requer um maior tempo para realização das análises.

Ares et al. (2011) compararam os resultados de quatro metodologias descritivas para avaliar sucos em pó comerciais sabor laranja e a dificuldade percebida pelos consumidores, nas diferentes técnicas. As metodologias estudadas foram sorting, mapeamento projetivo, escalas de intensidade e CATA. Cada técnica foi avaliada por um grupo de 50 consumidores. Além da descrição dos produtos, foi pedido aos consumidores que avaliassem a dificuldade durante a realização dos testes em uma escala estruturada de $9 \mathrm{~cm}$, ancorada com os termos "muito fácil" à esquerda e "muito difícil" à direita. Os resultados mostraram que as quatro metodologias foram capazes de identificar diferenças nas características sensoriais das bebidas de forma bastante semelhante (RV > 0,73; p-valor não apresentado). No entanto, o CATA e as escalas de intensidade foram consideradas mais fáceis de serem realizadas pelos consumidores. Segundo os autores, embora estas metodologias tenham aplicabilidade, devem ser utilizadas como complemento das análises descritivas realizadas com avaliadores treinados, no desenvolvimento de novos produtos. Além disso, devem ser analisadas cuidadosamente as diferenças individuais do consumidor, em análises futuras.

Cadena et al. (2014) compararam três metodologias rápidas - CATA, mapeamento projetivo e PSP - com a análise descritiva quantitativa, para caracterização sensorial de iogurtes funcionais com baixo teor de gordura enriquecidos com probióticos e prebióticos. Uma equipe de nove avaliadores treinados avaliou as amostras utilizando análise descritiva, enquanto três grupos de 81 consumidores utilizaram as três metodologias rápidas. Estas forneceram informações semelhantes sobre as principais diferenças entre as amostras. No entanto, a metodologia CATA forneceu uma configuração sensorial mais semelhante à fornecida pela $A D Q(R V=0,95$; $p$-valor não apresentado), enquanto que o mapeamento projetivo, a menos semelhante ( $R V=0,54$; p-valor não apresentado). As três metodologias também diferiram na sua capacidade de detectar diferenças entre as formulações (amostras) e na estabilidade das configurações das amostras.

Fleming et al. (2015) compararam três técnicas de perfil sensorial com consumidores (CATA, sorting e PSP), utilizando 10 compostos adstringentes. Todos os métodos mostraram-se viáveis para caracterização sensorial rápida de compostos adstringentes, uma vez que apresentaram mapas sensoriais visual e estatisticamente muito semelhantes (coeficiente RV normalizado maior que $5,00)$. Com base no estudo, os autores recomendaram a utilização do CATA para orientar a seleção dos pólos a serem utilizados no estudo de posicionamento sensorial polarizado (PSP).

Lazo et al. (2016) avaliaram 23 espécies diferentes de peixes, utilizando os métodos CATA e Perfil Livre. Dezoito avaliadores treinados com perfil semelhante foram divididos aleatoriamente em dois grupos. Um grupo avaliou as espécies de peixes utilizando CATA e o outro, usando Perfil Livre. Apesar de ambas as metodologias gerarem um número importante de descritores sensoriais para os produtos testados, a metodologia CATA apresentou um desempenho melhor do que o Perfil Livre, em termos de capacidade descritiva e discriminativa. De acordo com os coeficientes ( $R V>0,70)$, ambos os métodos proporcionaram uma localização similar dos produtos no espaço multidimensional.

Antúnez et al. (2017) compararam três metodologias descritivas baseadas na percepção do consumidor com a análise descritiva clássica, considerando, como estudo de caso, quatro conjuntos de bebidas em pó, diferindo no número de amostras e na extensão em que representavam toda a categoria do produto. A equipe de avaliadores treinados avaliou os conjuntos de amostras usando análise descritiva clássica, enquanto grupos de 100 consumidores utilizaram uma das três metodologias descritivas baseadas na percepção do consumidor: CATA, mapeamento projetivo e posicionamento sensorial polarizado (PSP). Nos quatro conjuntos de amostras, as metodologias baseadas na percepção dos consumidores forneceram informações semelhantes sobre as semelhanças e diferenças marcantes entre as amostras, e não diferiram grandemente das informações obtidas pela análise descritiva. A principal diferença entre as metodologias foi relacionada à importância relativa dada às características sensoriais na avaliação das diferenças entre amostras, o que levou a diferenças na dimensionalidade dos espaços sensoriais. As configurações de amostras necessárias para explicar completamente as diferenças entre amostras exigiram mais dimensões no mapeamento projetivo e no PSP do que na análise descritiva e no CATA. Nenhuma das metodologias baseadas no consumidor superou a análise descritiva em termos de capacidade de discriminar amostras, sendo o CATA a metodologia que apresentou a menor discriminação.

\section{Conclusão}

Os métodos apresentados vêm fornecendo configurações sensoriais semelhantes às obtidas pela análise descritiva convencional e são eficientes na discriminação de amostras, mesmo utilizando consumidores na avaliação. Entretanto, não podem substituir a precisão da análise descritiva clássica, uma vez que os avaliadores 
Metodologias sensoriais descritivas mais rápidas e versáteis - uma atualidade na ciência sensorial Alcantara, M.; Freitas-Sá, D. G. C.

são extensivamente treinados na identificação e na quantificação de atributos sensoriais claramente definidos. Por permitirem realizar caracterizações sensoriais com consumidores, são úteis para revelar a percepção dos consumidores acerca dos produtos, utilizando seu próprio vocabulário, fornecendo informações valiosas durante o desenvolvimento de novos produtos ou na concepção de campanhas de marketing.

A seleção de uma nova metodologia deve levar em consideração o tempo e os recursos disponíveis para sua implementação, o objetivo do estudo, o tipo de avaliador, bem como questões práticas, como a facilidade em executar as tarefas solicitadas, o número de amostras e as características inerentes ao tipo de produto avaliado (por exemplo, produtos que requerem controle cuidadoso da temperatura ou que tenham características sensoriais intensas e persistentes). O uso de consumidores favorece a aplicação de metodologias simples, como o questionário CATA, enquanto que, se há uma equipe de avaliadores treinados disponível, as características sensoriais dos produtos podem ser obtidas de maneira mais rápida, através de técnicas como Perfil Flash, sorting, mapeamento projetivo ou PSP, sendo o Perfil Flash considerado o mais demorado e responsável por uma maior saturação, por parte dos avaliadores.

Destaca-se que, para determinar pequenas diferenças na intensidade de atributos específicos, a análise descritiva clássica continua sendo a melhor opção para a caracterização sensorial de produtos.

\section{Referências}

ABDI, H. RV coefficient and congruence coefficient. In: SALKIND, N. J. (Ed.). Encyclopedia of measurement and statistics. Thousand Oaks, CA: Sage, 2007a. p. 849-853.

ABDI, H. Metric multidimensional scaling. In: SALKIND, N. J. (Ed.). Encyclopedia of measurement and statistics. Thousand Oaks, CA: Sage, 2007b. p. 598-605.

ABDI, H.; WILLIAMS, L. J. Principal component analysis. Wiley Interdisciplinary Reviews: Computational Statistics, v. 2, n. 4, p. 433-459, 2010. http://dx.doi.org/10.1002/wics.101.

ABDI, H.; WILLIAMS, L. J.; VALENTIN, D. Multiple factor analysis: principal component analysis for multitable and multiblock data sets. Wiley Interdisciplinary Reviews: Computational Statistics, v. 5, n. 2, p. 149-179, 2013. http://dx.doi.org/10.1002/wics.1246.

ALBERT, A.; VARELA, P.; SALVADOR, A.; HOUGH, G.; FISZMAN, $S$. Overcoming the issues in the sensory description of hot served food with a complex texture. Application of QDA_, flash profiling and projective mapping using panels with different degrees of training. Food Quality and Preference, v. 22, n. 5 , p. 463-473, 2011.
ANTÚNEZ, A.; SALVADOR, A.; DE SALDAMANDO, L.; VARELA, P.; GIMÉNEZ, A.; ARES, G. Evaluation of data aggregation in Polarized Sensory Positioning. Journal of Sensory Studies, V. 30, n. 1, p. 46-55, 2015. http://dx.doi.org/10.1111/joss.12135.

ANTÚNEZ, L.; ARES, G.; GIMÉNEZ, A.; JAEGER, S. R. Do individual differences in visual attention to CATA questions affect sensory product characterization? A case study with plain crackers. Food Quality and Preference, v. 48, Part A, p. 185-194, 2016.

ANTÚNEZ, L.; VIDAL, L.; SALDAMANDO, L.; GIMÉNEZ, A.; ARES, G. Comparison of consumer-based methodologies for sensory characterization: case study with four sample sets of powdered drinks. Food Quality and Preference, v. 56, Part A, p. 149-163, 2017

ARES, G.; ANDRADE, J. C.; ANTÚNEZ, L.; ALCAIRE, F.; SWANEYSTUEVE, M.; GORDON, S.; JAEGER, S. R. Hedonic product optimisation: CATA questions as alternatives to JAR scales. Food Quality and Preference, v. 55, p. 67-78, 2017.

ARES, G.; ANTÚNEZ, L.; GIMENEZ, A.; JAEGER, S. R. List length has little impact on consumers' visual attention to CATA questions. Food Quality and Preference, v. 42, p. 100-109, 2015a.

ARES, G.; ANTÚNEZ, L.; OLIVEIRA, D.; ALCAIRE, F.; GIMÉNEZ, A.; BERGET, I.; N/ES, T.; VARELA, P. Pole selection in polarized sensory positioning: insights from the cognitive aspects behind the task. Food Quality and Preference, v. 46, p. 48-57, 2015b.

ARES, G.; JAEGER, S. R.; ANTÚNEZ, L.; VIDAL, L.; GIMÉNEZ, A.; COSTE, B.; PICALLO, A.; CASTURA, J. C. Comparison of TCATA and TDS for dynamic sensory characterization of food products. Food Research International, v. 78, p. 148-158, 2015c. PMid:28433276. http://dx.doi.org/10.1016/j.foodres.2015.10.023.

ARES, G.; REIS, F.; OLIVEIRA, D.; ANTÚNEZ, L.; VIDAL, L.; GIMENEZ, A.; CHHEANG, S. L.; HUNTER, D. C.; KAMD, K.; ROIGARD, C. M.; PAISLEY, A. G.; BERESFORD, M. K.; JIN, D.; JAEGER, S. R. Recommendations for use of balanced presentation order of terms in CATA questions. Food Quality and Preference, v. 46, p. 137-141, 2015d.

ARES, G.; ANTÚNEZ, L.; GIMENEZ, A.; ROIGARD, C. M.; PINEAU, B.; HUNTER, D. C.; JAEGER, S. R. Further investigations into the reproducibility of check-all-that-apply (CATA) questions for sensory product characterization elicited by consumers. Food Quality and Preference, v. 36, p. 111-121, 2014a.

ARES, G.; BRUZZONE, F.; VIDAL, L.; CADENA, R. S.; GIMÉNEZ, A.; PINEAU, B.; HUNTER., D. C.; PAISLEY, A. G.; JAEGER, S. R. Evaluation of a rating-based variant of check-all-that-apply questions: rate-all-that-apply (RATA). Food Quality and Preference, v. 36, p. 87-95, 2014b.

ARES, G.; VARELA, P. Comparison of novel methodologies for sensory characterization. In VARELA, P.; ARES, G. Novel techniques in sensory characterization and consumer profiling. Boca Raton: CRC Press, 2014. chap. 14, p. 365-389. 
Metodologias sensoriais descritivas mais rápidas e versáteis - uma atualidade na ciência sensorial Alcantara, M.; Freitas-Sá, D. G. C.

ARES, G.; BARREIRO, C., DELIZA, R.; GIMÉNEZ, A.; GÁMBARO, A. Application of a check-all-that-apply question to the development of chocolate Milk desserts. Journal of Sensory Studies, v. 25, n. 1, p. 67-86, 2010.

ARES, G.; JAEGER, S. R. Check-all-that-apply questions: Influence of attribute order on sensory product characterization. Food Quality and Preference, v. 28, n. 1, p. 141-153, 2013. http:// dx.doi.org/10.1016/j.foodqual.2012.08.016.

ARES, G.; VARELA, P.; RADO, G.; GIMÉNEZ, A. Are consumer profiling techniques equivalent for some product categories? The case of orange-flavoured powdered drinks. International Journal of Food Science \& Technology, v. 46, n. 8, p. 16001608, 2011. http://dx.doi.org/10.1111/j.1365-2621.2011.02657.x.

ASSOCIAÇÃO BRASILEIRA DE NORMAS TÉCNICAS - ABNT. ABNT NBR ISO 11132:2016 sensory analysis - methodology - guidelines for monitoring the performance of a quantitative sensory panel. Rio de Janeiro, 2016. 29 p.

BALLAY, S.; WARRENBURG, S.; SIEFFERMANN, J.-M.; GLAZMAN, L.; GAZANO, G. A new fragrance language: intercultural knowledge and emotions. In: 24th IFSCC Congress - Integration of Cosmetic Sciences, 24., 16-19 oct. 2006, Osaka. Proceedings... Osaka, Japan: IFSCC, 2006.

BARCENAS, P.; ELORTONDO, F. J. P.; ALBISU, M. Projective mapping in sensory analysis of ewes milk cheeses: a study on consumers and trained panel performance. Food Research International, v. 37, n. 7, p. 723-724, 2004. http://dx.doi. org/10.1016/j.foodres.2004.02.015.

BRUZZONE, F.; ARES, G.; GIMÉNEZ, A. Consumers' texture perception of milk desserts. II - comparison with trained assessors' data. Journal of Texture Studies, v. 43, n. 3, p. 214-226, 2012. http://dx.doi.org/10.1111/j.1745-4603.2011.00332.x.

CADENA, R. S.; CAIMI, D.; JAUNARENA, I.; LORENZO, I.; VIDAL, L.; ARES, G.; DELIZA, R.; GIMÉNEZ, A. Comparison of rapid sensory characterization methodologies for the development of functional yogurts. Food Research International, v. 64, p. 446-455, 2014. http://dx.doi.org/10.1016/j.foodres.2014.07.027.

CARTIER, R.; RYTZ, A.; LECOMTE, A.; POBLETE, F.; KRYSTLIK, J.; BELIN, E.; MARTIN, N. Sorting procedure as an alternative to quantitative descriptive analysis to obtain a product sensory map. Food Quality and Preference, v. 17, n. 7-8, p. 562-571, 2006.

CASTURA, J. C.; ANTÚNEZ, L.; GIMÉNEZ, A.; ARES, G. Temporal Check-All-That-Apply (TCATA): a novel dynamic method for characterizing products. Food Quality and Preference, v. 47, parte A, p. 79-90, 2016.

CASTURA, J. C.; MEYNERS, M. Check-All-That-Apply questions. In: ARES, G.; VARELA, P. Novel techniques in sensory characterization and consumer profiling. Boca Raton: CRC Press, 2014. chap. 11, p. 271-305.

CHOLLET, S.; LELIÈVRE, M.; ABDI, H.; VALENTIN, D. Sort and beer: everything you wanted to know about the sorting task but did not dare to ask. Food Quality and Preference, v. 22, n. 6, p. 507-520, 2011.

CHOLLET, S.; VALENTIN, D.; ABDI, H. Free sorting task. In: ARES, G.; VARELA, P. Novel techniques in sensory characterization and consumer profiling. Boca Raton: CRC Press, 2014. chap. 8, p. 207-228.

COXON, A. P. M. Sorting data: collection and analysis (Sage University Papers Series on Quantitative Applications in the Social Science, p. 7-127). Thousand Oaks: Sage, 1999.

DAIROU, V.; SIEFFERMANN, J. M. A comparison of 14 jams characterized by conventional profile and a quick original method, the Flash Profile. Journal of Food Science, v. 67, n. 2, p. 826-834, 2002. http://dx.doi.org/10.1111/j.1365-2621.2002.tb10685.x.

DE SALDAMANDO, L.; DELGADO, J.; HERENCIA, P.; GIMÉNEZ, A.; ARES, G. Polarized sensory positioning: do conclusions depend on the poles? Food Quality and Preference, v. 29, n. 1, p. 25-32, 2013.

DEHLHOLM, C. Descriptive sensory evaluations: comparison and applicability of novel rapid methodologies. Copenhagen, Denmark: SL grafik, 2012.

DEHLHOLM, C. Projective Mapping and Napping. In: ARES, G.; VARELA, P. Novel techniques in sensory characterization and consumer profiling. Boca Raton: CRC Press, 2014. chap. 9, p. 229-254.

DEHLHOLM, C.; BROCKHOFF, P. B.; MEINERT, L.; AASLYNG, M. D.; BREDIE, W. L. P. Rapid descriptive sensory methodsComparison of free multiple sorting, partial napping, napping, flash profiling and conventional profiling. Food Quality and Preference, v. 26, n. 2, p. 267-277, 2012.

DELARUE, J. Flash Profile. In: ARES, G.; VARELA, P. Novel techniques in sensory characterization and consumer profiling. Boca Raton: CRC Press, 2014. chap. 7, p. 175-205.

DELARUE, J.; SIEFFERMANN, J. M. Sensory mapping using Flash Profile. Comparison with a conventional descriptive method for the evaluation of the flavour of fruit dairy products. Food Quality and Preference, v. 15, n. 4, p. 383-392, 2004.

DIJKSTERHUIS, G. B.; GOWER, J. C. The interpretation of generalized procrustes analysis and allied methods. Food Quality and Preference, v. 3, n. 2, p. 67-87, 1991.

DOOLEY, L.; LEE, Y. S.; MEULLENET, J. F. The application of check-all-that-apply (CATA) consumer profiling to preference mapping of vanilla ice cream and its comparison to classical external preference mapping. Food Quality and Preference, v. 21, p. 394-401, 2010.

ESCOFIER, B.; PAGÈS, J. Analyses factorielles simples et multiples, objectifs méthodes et interprétation. 4th ed. Paris: Dunod, 2008. 241 p.

FLEMING, E. E.; ZIEGLER, G. R.; HAYES, J. E. Check-all-thatapply (CATA), sorting, and polarized sensory positioning (PSP) 
Metodologias sensoriais descritivas mais rápidas e versáteis - uma atualidade na ciência sensorial Alcantara, M.; Freitas-Sá, D. G. C.

with astringent stimuli. Food Quality and Preference, v. 45, p. 41-49, 2015.

GOWER, J. C. Generalized procrustes analysis. Psychometrika, v. 40, n. 1, p. 33-51, 1975. http://dx.doi.org/10.1007/BF02291478.

HOPFER, H.; HEYMANN, H. A summary of projective mapping observations - the effect of replicates and shape, and individual performance measurements. Food Quality and Preference, v. 28, n. 1, p. 164-181, 2013.

HUGHSON, A. L.; BOAKES, R. A. The knowing nose: the role of knowledge in wine expertise. Food Quality and Preference, v. 13, n. 7-8, p. 463-472, 2002.

JAEGER, S. R.; BERESFORD, M. K.; PAISLEY, A. G.; ANTÚNEZ, L.; VIDAL, L.; CADENA, R. S.; GIMÉNEZ, A.; ARES, G. Check-allthat-apply (CATA) questions for sensory product characterization by consumers: Investigations into the number of terms used in CATA questions. Food Quality and Preference, v. 42, p. 154-164, 2015.

JAEGER, S. R.; FISZMAN, S.; REIS, F.; CHHEANG, S. L.; KAM, K.; PINEAU, B.; DELIZA, R.; ARES, G. Influence of evoked contexts on hedonic product discrimination and sensory characterizations using CATA questions. Food Quality and Preference, v. 56, p. 138-148, 2017. Part A.

JAEGER, S.; LEANG CHHEANG, S.; YIN, J.; BAVA, C. M.; GIMENEZ, A.; VIDAL, L.; ARES, G. Check-all-that-apply (CATA) responses elicited by consumers: within-assessor reproducibility and stability of sensory product characterizations. Food Quality and Preference, v. 30, n. 1, p. 56-67, 2013.

KENNEDY, J.; HEYMANN, H. Projective mapping and descriptive analysis of milk and dark chocolates. Journal of Sensory Studies, v. 24, n. 2, p. 220-233, 2009. http://dx.doi.org/10.1111/j.1745459X.2008.00204.x.

KING, M. C.; CLIFF, M. A.; HALL, J. W. Comparison of projective mapping and sorting data collection and multivariate methodologies for identification of similarity-of-use of snack bars. Journal of Sensory Studies, v. 13, n. 3, p. 347-358, 1998. http://dx.doi. org/10.1111/j.1745-459X.1998.tb00094.X.

KOBAYASHI, M. L.; BENASSI, M. T. Caracterização sensorial de cafés solúveis comerciais por Perfil Flash. Semina: Ciências Agrárias, v. 33, p. 3081-3092, 2012. Suplemento 2.

LADO, J.; VICENTE, E.; MANZZIONI, V.; ARES, G. Application of a check-all-that-apply question for the evaluation of strawberry cultivars from a breeding program. Journal of the Science of Food and Agriculture, v. 90, n. 13, p. 2268-2275, 2010. PMid:20648524. http://dx.doi.org/10.1002/jsfa.4081.

LAWLESS, H. T.; HEYMANN, H. Sensory evaluation of food: principles and practices. Gaithersburg: Aspen, 1999.

LAWLESS, H. T.; HEYMANN, H. Sensory evaluation of food: principles and practices. New York: Springer, 2010.
LAZO, O.; CLARET, A.; GUERRERO, L. A comparison of two methods for generating descriptive attributes with trained assessors: check-all-that-apply (CATA) vs. free choice profiling (FCP). Journal of Sensory Studies, v. 31, n. 2, p. 163-176, 2016. http://dx.doi.org/10.1111/joss.12202.

LELIĖVRE, M.; CHOLLET, S.; ABDI, H.; VALENTIM, D. What is the validity of the sorting task for describing beers? A study using trained and untrained assessors. Food Quality and Preference, v. 19, n. 8, p. 697-703, 2008.

MEYNERS, M. Temporal liking and CATA analysis of TDS data on flavored fresh cheese. Food Quality and Preference, v. 47, p. 101-108, 2016. Part A.

MEYNERS, M.; CASTURA, J. C. Randomization of CATA attributes: Should attribute lists be allocated to assessors or to samples? Food Quality and Preference, v. 48, p. 210-215, 2016.

MONTANUCI, F. D.; MARQUES, D. R.; MONTEIRO, A. R. G. Flash Profile for rapid descriptive analysis in sensory characterization of passion fruit juice. Acta Scientiarum Technology, v. 37, n. 3, p. 337-344, 2015.

MOUSSAOUI, K.; VARELA, P. Exploring consumer product profiling techniques and their linkage to a quantitative descriptive analysis. Food Quality and Preference, v. 21, n. 8, p. 1088-1099, 2010.

NESTRUD, M. A.; LAWLESS, H. T. Perceptual mapping of apples and cheeses using projective mapping and sorting. Journal of Sensory Studies, v. 25, n. 3, p. 390-405, 2010. http://dx.doi. org/10.1111/j.1745-459X.2009.00266.x.

OLIVEIRA, A. P. V.; BENASSI, M. T. Perfil Livre: uma opção para análise sensorial descritiva. Boletim da Sociedade Brasileira de Ciência e Tecnologia de Alimentos, v. 37, p. 66-72, 2003.

PAGÈS, J. Collection and analysis of perceived product interdistances using multiple factor analysis: application to the study of 10 white wines from the Loire Valley. Food Quality and Preference, v. 16, n. 7, p. 642-649, 2005.

PERRIN, L.; SYMONEAUX, R.; MAÎTRE, I.; ASSELIN, C.; JOURJON, F.; PAGÈS, J. Comparison of three sensory methods for use with the Napping procedure: case of ten wines from Loire valley. Food Quality and Preference, v. 19, n. 1, p. 1-11, 2008.

REINBACH, H. C. GIACALONE, D.; RIBEIRO, L. M.; BREDIE, W. L. P.; FRØST, M. B. Comparison of three sensory profiling methods based on consumer perception: CATA, CATA with intensity and Napping $\AA$. Food Quality and Preference, v. 32 , p. 160-166, 2014. part B.

RISVIK, E.; MCEWAN, J. A.; COLWILL, J. S.; ROGERS, R.; LYON, D. H. Projective mapping: a tool for sensory analysis and consumer research. Food Quality and Preference, v. 5, p. 263-269, 1994.

RISVIK, E.; MCEWAN, J. A.; RODBOTTEN, M. Evaluation of sensory profiling and projective mapping data. Food Quality and Preference, v. 8, p. 63-71, 1997. 
Metodologias sensoriais descritivas mais rápidas e versáteis - uma atualidade na ciência sensorial

Alcantara, M.; Freitas-Sá, D. G. C.

RUARK, A.; VINGERHOEDS, M. H.; KREMER, S.; NIJENHUIS-DE VRIES, M. A.; PIQUERAS-FISZMAN, B. Insights on older adults' perception of at-home sensory-hedonic methods: a case of Ideal Profile Method and CATA with ideal. Food Quality and Preference, v. 53, p. 29-38, 2016.

SANTOSA, M.; ABDI, H.; GUINARD, J. A modified sorting task to investigate consumer perceptions of extra virgin olive oils.

Food Quality and Preference, v. 21, n.7, p. 881-892, 2010.

STONE, H.; SIDEL, J. L. Sensory evaluation practices. 3. ed. New York: Academic Press, 2004. 377 p.

TAREA, S.; CUVELIER, G.; SIEFFERMANN, J. M. Sensory evaluation of the texture of 49 commercial apple and pear purees. Journal of Food Quality, v. 30, n. 6, p. 1121-1131, 2007.

TEILLET, E.; SCHLICH, P.; URBANO, C.; CORDELLE, S.; GUICHARD, E. Sensory methodologies and the taste of water. Food Quality and Preference, v. 21, n. 8, p. 967-976, 2010.

TEILLET, E. Polarized sensory positioning methodologies. In VARELA, P.; ARES, G. Novel techniques in sensory characterization and consumer profiling. Boca Raton: CRC Press, 2014. chap. 10, p. 255-270.

TERHAAG, M. M.; BENASSI, M. T. Perfil Flash: uma opção para análise descritiva rápida. Brazilian Journal and Food Technology, p. 140-151, 2010. 6 SENSIBER, 19-21 ago. 2010.

VALENTIN, D.; CHOLLET, S.; LELIÈVRE, M.; ABDI, H. Quick and dirty but still pretty good: a review of new descriptive methods in food science. International Journal of Food Science \&
Technology, v. 47, n. 8, p. 1563-1578, 2012. http://dx.doi. org/10.1111/j.1365-2621.2012.03022.x.

VARELA, P.; ARES, G. Sensory profiling, the blurred line between sensory and consumer science. A review of novel methods for product characterization. Food Research International, v. 48, n. 2 , p. 893-908, 2012. http://dx.doi.org/10.1016/j.foodres.2012.06.037.

VARELA, P.; SVARTEBEKK MYHRER, K.; NÆS, T.; HERSLETH, M. The best of both worlds: rapidity and enhanced discrimination with a trained panel. Exploration of global and partial PSP with a descriptive step. In: 6th EUROPEAN CONFERENCE ON SENSORY AND CONSUMER RESEARCH, 6., 7-10 sept. 2014, Copenhaguen. Proceedings... Copenhaguen, Denmark, 2014.

VIDAL, L.; ARES, G.; HEDDERLEY, D. I.; MEYNERS, M.; JAEGER, S. R. Comparison of rate-all-that-apply (RATA) and check-allthat-apply (CATA) questions across seven consumer studies. Food Quality and Preference, 2016. In press. http://dx.doi. org/10.1016/j.foodqual.2016.12.013.

VIDAL, L.; CADENA, R. S.; ANTÚNEZ, L.; GIMÉNEZ, A.; VARELA, P.; ARES, G. Stability of sample configurations from projective mapping: how many consumers are necessary? Food Quality and Preference, v. 34, p. 79-87, 2014. http://dx.doi.org/10.1016/j. foodqual.2013.12.006.

WILLIAMS, A. A.; LANGRON, S. P. The use of free-choice profiling for the evaluation of commercials ports. Journal of the Science of Food and Agriculture, v. 35, n. 5, p. 558-568, 1984. http:// dx.doi.org/10.1002/jsfa.2740350513. 